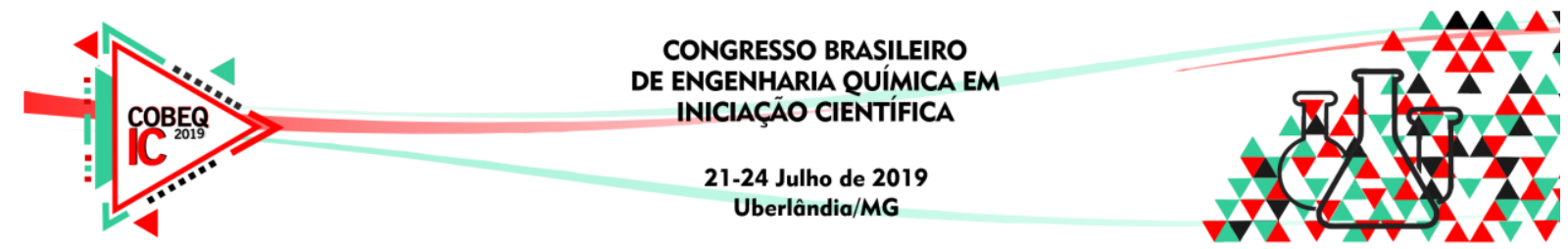

\title{
DIMENSIONAMENTO DE UMA COLUNA DE ABSORÇÃO POR SOLUÇÕES AMINAS EM ESCALA PILOTO PARA CAPTURA DE $\mathrm{CO}_{2}$
}

\author{
M. C. S. SILQUEIRA ${ }^{1}$, J. F. C. FILHO ${ }^{2}$, K.I. REZENDE ${ }^{3}$, B. W. M. $\operatorname{SILVA}^{4}$ e S. N. \\ RABELO $^{5}$ \\ ${ }^{1}$ Universidade de Itaúna, Faculdade de Engenharia Mecânica \\ ${ }^{2}$ Universidade de Itaúna, Faculdade de Engenharia Mecânica \\ ${ }^{3}$ Universidade de Itaúna, Faculdade de Engenharia Mecânica \\ ${ }^{4}$ Universidade de Itaúna, Faculdade de Engenharia Mecânica \\ ${ }^{5}$ Universidade de Itaúna, Faculdade de Engenharia Mecânica e Universidade Federal de \\ Minas Gerais, Departamento de Engenharia Mecânica \\ E-mail para contato: sasanogueirarab@hotmail.com
}

\begin{abstract}
RESUMO - A captura de $\mathrm{CO}_{2}$ é motivada pelo aumento significativo da concentração desse gás na atmosfera, principalmente devido ao rápido crescimento industrial mundial. Além disso, o $\mathrm{CO}_{2}$ é um dos principais gases contribuintes do efeito estufa. Um dos principais métodos de captura desse gás é por meio da absorção por aminas. Dessa forma, o presente trabalho objetiva realizar o dimensionamento de uma coluna de absorção feita em vidro em escala piloto com recheio estruturado para captura de $\mathrm{CO}_{2}$ por meio de soluções aquosas de aminas operando à pressão atmosférica. O dimensionamento otimiza o processo de absorção à fim de evitar inundações e elevadas quedas de pressão na coluna. Para o dimensionamento da coluna, uma sequência de avaliação é desenvolvida: (1) seleção da estrutura contatora, que otimizará o contato entre as fases líquida e gasosa; (2) análise das condições de operação; (3) cálculo da altura da coluna baseada no cálculo da taxa de transferência de massa; (4) cálculo do diâmetro da coluna tendo como base as vazões de solvente e do gás, e considerações hidráulicas. A partir dessa sequência, a coluna de absorção foi dimensionada para operar com diferentes tipos de soluções de aminas, utilizadas em escala laboratorial.
\end{abstract}

\section{INTRODUÇÃO}

O processo de absorção baseia-se na transferência de um componente da fase gasosa para a líquida, na qual ele é solúvel. A absorção é, sem dúvida, a operação de purificação de gases mais importante e mais utilizada em larga escala industrial. Segundo Kohl e Nielsen (1997) o processo de absorção pode ser classificado baseado na interação entre solvente e soluto em solução física em reação reversível e irreversível. 


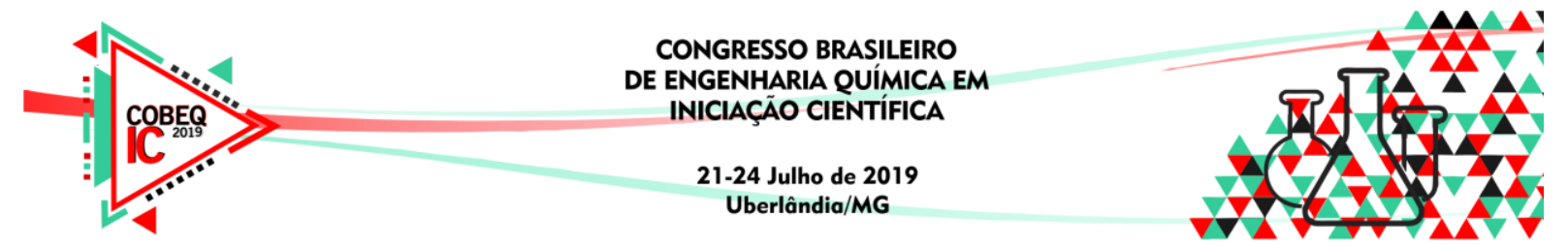

No processo de absorção por reação química em um processo reversível, o dimensionamento da coluna de absorção é fator de extrema importância para o processo. $\mathrm{O}$ tamanho da coluna é fundamental para se obter uma ótima interação entre os componentes, reduzir as quedas de pressão e maximizar o rendimento do processo (AROONWILAS, 2001).

Desta forma, devido à relevância do dimensionamento de coluna para um processo de absorção reversível, o presente trabalho apresenta uma análise de dimensionamento para uma coluna de recheio de absorção para captura do $\mathrm{CO}_{2}$ em escala laboratorial.

\section{METODOLOGIA}

O dimensionamento de uma coluna de absorção obedece a uma sequência lógica (KOHL E NIELSEN, 1997). Inicia-se com a escolha do solvente que será empregado na coluna, para que assim o design da coluna possa ser estabelecido. Para fins desse estudo, selecionaram-se soluções como as alcanolaminas (MEA, monoetanolamina), amino-2-metil1-propanol (AMP) e piperazina (PZ), uma vez que esses componentes apresentam elevada capacidade de absorção de $\mathrm{CO}_{2}$. Assim, o dimensionamento da estrutura da coluna de absorção e seus componentes foram realizados de acordo com as etapas que são abordadas adiante.

\subsection{Seleção Da Estrutura Contatora}

De acordo com Kohl e Nielsen (1997) as estruturas contatoras que ocupam toda a seção transversal da torre da coluna de absorção possuem a função de promover uma extensa área de contato entre as fases líquida e gasosa, o que favorece a transferência de massa. Essas estruturas permitem melhor distribuição do líquido na coluna de modo que o gás a ser tratado tenha contato com a maior quantidade de solução possível.

Para formar essas estruturas contadoras em colunas de absorção, existem dois tipos principais de recheios: os randômicos e os estruturados (KOHL E NIELSEN, 1997). O primeiro tipo, é caracterizado por ser distribuído em várias unidades de forma aleatória na torre, enquanto o segundo pode ser definido como blocos compactos com estrutura bem definida que permitem uma melhor distribuição do líquido.

Estudos apontam que recheios randômicos e estruturados, geralmente, apresentam uma elevada área por unidade de volume, garantindo uma melhor troca de massa e baixa perda de carga, o que é vital para redução no consumo de energia (MELLO, 2013). Dessa forma, o recheio selecionado para realizar-se o dimensionamento da coluna foi o recheio estruturado pelo maior desempenho comparado ao recheio randômico em colunas de pequena escala (Kohl e Nielsen, 1997)

Os recheios estruturados são basicamente constituídos de lâminas metálicas cofiguradas com pequenas laminas de pequena espessura dispostas verticalmente na direção do fluxo ascendente da corrente gasosa. Estas são ranhuradas, perfuradas e possuem inclinação de $45^{\circ}$, de modo que o líquido escoe pelas ranhuras pela gravidade, e forme uma fina lamina, facilitado a troca de massas.( Nasser Junior, 2009) 


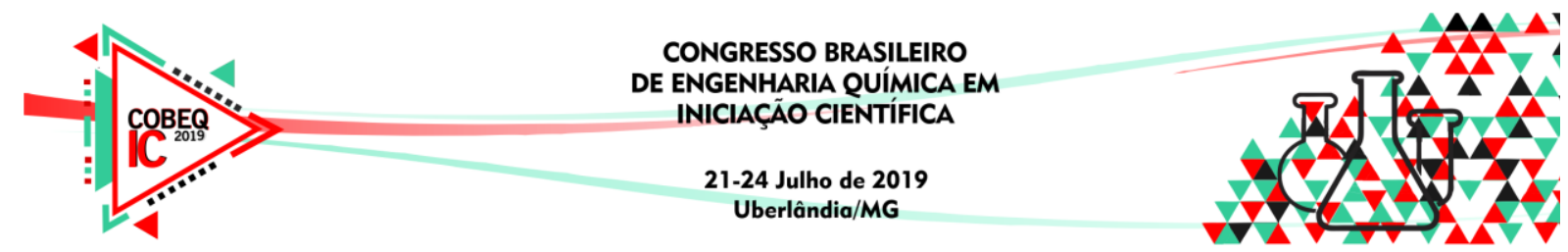

FIGURA 1 - Exemplo de recheio estrutrado "Mellapak 250 Y/X"

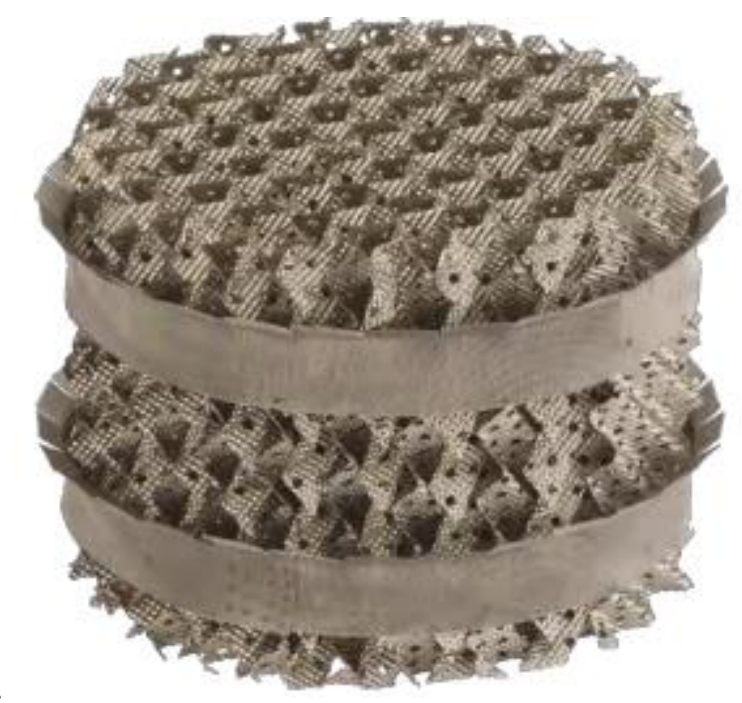

Fonte: Sulzer Chemtech, 2018

\section{Análise Das Condições De Operação}

O cálculo da altura da coluna baseia-se nos balanços de massa e energia, da força motriz para transporte de massa e dos coeficientes de transporte de massa. Enquanto o diâmetro da coluna, depende da vazão de gás, suas propriedades e da razão entre as vazões de líquido e gás (L/V). Já a altura e o volume total do recheio dependem da magnitude das concentrações desejadas e das velocidades de transferência de massa por unidade de volume de recheio (MELLO, 2013).

A FIGURA 1 apresenta um esquema para estimativa do balanço de massa da coluna de absorção contracorrente. As correntes de entrada são as vazões do gás rico em soluto e do líquido pobre em soluto; as correntes de saída são vazões de gás pobre em soluto e do líquido rico em soluto.

FIGURA 1 - Representação de um volume de controle.

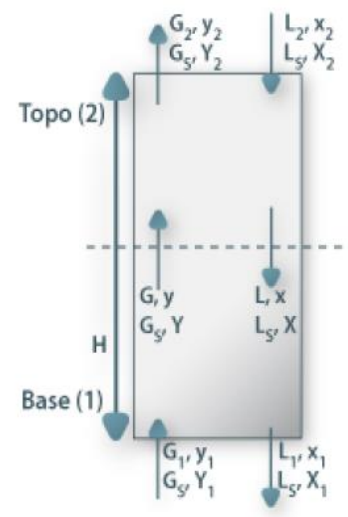




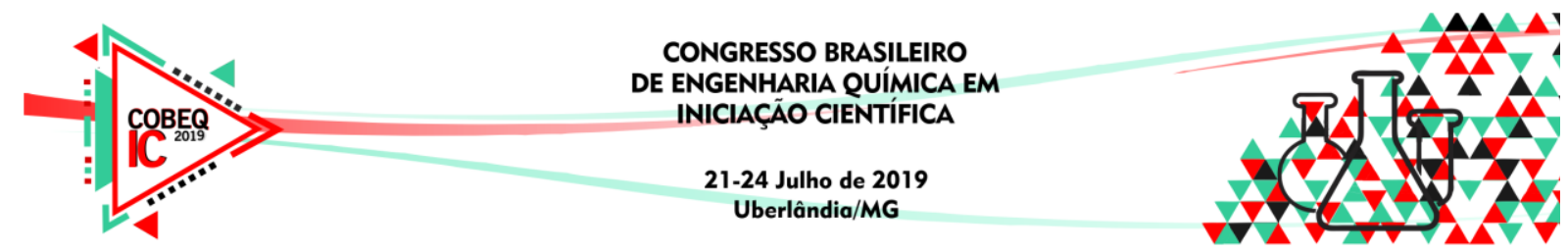

Fonte: Adaptado de Separações e operações unitárias (2018).

Conforme observa-se nas Equações 1 e 2 aplicando-se um balanço molar entre a base e uma seção genérica da coluna, tem-se que:

Mols de soluto na entrada = mols de soluto na saída

$\mathrm{L}_{\mathrm{x}}+\mathrm{G}_{1} \cdot \mathrm{y}_{1}+\mathrm{L}_{1 . \mathrm{X}_{1}}$

Aplicando-se balanço de razão molar em correntes isentas de soluto para obtenção da linha de operação, obtêm-se as Equações 3, 4, 5 e 6:

$$
\begin{aligned}
& \text { Ls. }\left(\frac{x}{1-x}\right)+G s \cdot\left(\frac{y 1}{1-y 1}\right)=L s \cdot\left(\frac{x 1}{1-x 1}\right)+G s\left(\frac{y}{1-y}\right) \\
& \text { Ls. } \mathrm{X}+\mathrm{Gs} . \mathrm{Y} 1=\mathrm{Ls} . \mathrm{X} 1+\mathrm{Gs} . \mathrm{Y} \\
& \mathrm{Gs}(\mathrm{Y} 1-\mathrm{Y})=\mathrm{Ls}(\mathrm{X} 1-\mathrm{X}) \\
& \mathrm{Y}=\left(\frac{L s}{G s}\right) \cdot \mathrm{X}+\mathrm{Y} 1-\left(\frac{L s}{G s}\right) \cdot \mathrm{X} 1
\end{aligned}
$$

A linha de operação para uma coluna de absorção não é uma reta, pois a absorção do componente na fase gasosa diminui essa corrente quando escoa pela coluna enquanto a vazão de líquido aumenta.

Aplicando-se o balanço molar entre o topo e a base, tem-se uma relação entre as velocidades das fases gasosa e líquida, fornecendo a inclinação da linha de operação, de acordo com as Equações 7 e 8.

$$
\text { Ls. X2 + Gs. Y1 = Ls. X1 + Gs. Y2 }
$$

$$
\left(\frac{L s \text { mín }}{G s}\right)=\frac{(Y 1-Y 2)}{(X 1 m \text { áx }-X 2)}
$$

Deve-se ressaltar que o caudal de líquido não pode ser inferior a um valor limite (mínimo) correspondente à obtenção de condições de saturação na base da coluna $\left(X l_{\text {máx }}\right) \mathrm{em}$ equilíbrio com $Y 1$ ), conforme a Equação 9.

$$
\left(\frac{\operatorname{Ls}(\min )}{\mathrm{Gs}}\right)=\frac{(Y 1-Y 2)}{(X 1 \text { má } x-X 2)}
$$

Este será o caudal mínimo de líquido para se conseguir remover $G S(Y 1-Y 2) / \mathrm{mols}$ de soluto "A" por unidade de tempo, obtendo-se uma composição máxima na corrente líquida de saída. 


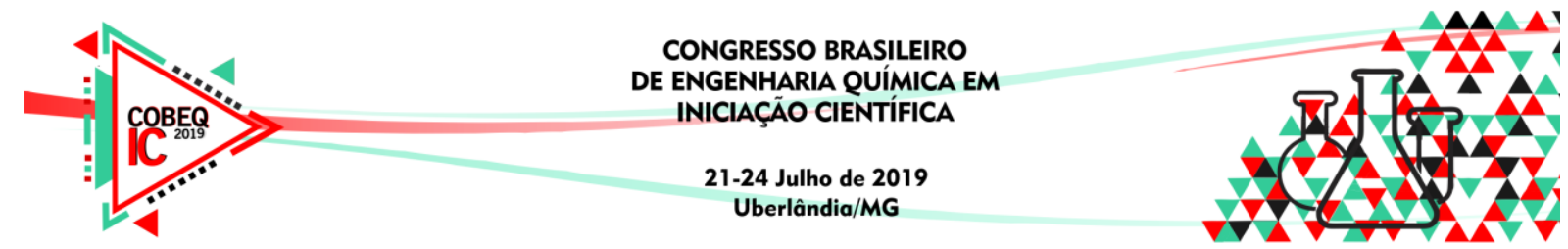

O projeto de uma coluna de absorção também tem como base o coeficiente global de transferência na fase gasosa. Por meio do balanço diferencial de soluto na fase gasosa, para uma vazão constante ao longo da coluna, tem-se que a quantidade de soluto absorvida na altura "dz" é igual a velocidade de absorção multiplicado pelo volume diferencial, conforme a Equação 10.

$$
- \text { V.dy }=\text { kg.a. }(y-y *) . S . d z
$$

Em que,

$\mathrm{S}=$ seção transversal da coluna $\left(\mathrm{m}^{2}\right), \mathrm{dz}=$ variação da altura da coluna $(\mathrm{m}), \mathrm{y}=$ fração molar da fase gasosa, $\mathrm{y}^{*}=$ fração molar de equilíbrio com a fase líquida, $\mathrm{Kg} . \mathrm{a}=$ coeficiente global de transferência de massa na fase gás $\left(\mathrm{kg} \cdot \mathrm{mol} / \mathrm{h} \cdot \mathrm{m}^{3}\right.$.atm).

Rearranjando-se a Equação 10, e considerando como constantes os termos Kg.a e V, têm-se que:

$\frac{\text { K.g.a.S }}{\mathrm{V}} \cdot \int d z=\int(\mathrm{dy}-\mathrm{y}-\mathrm{y} *$ ye. $\mathrm{ys})$

Logo, a altura da coluna pode ser determinada pela Equação 12:

$$
\mathrm{Zt}=\frac{\frac{\mathrm{v}}{\mathrm{s}}}{\mathrm{Kg} \cdot \mathrm{a}} \cdot \int_{y s}^{y e} \frac{d y}{y-y^{*}}
$$

Em que a integral da Equação 12 representa a variação da concentração no vapor dividida pela força motriz média, e é chamada de número de unidades de transferência (NUT). Já o primeiro termo da equação é conhecido como altura de uma unidade de transferência (HUT). Logo, obtém-se uma expressão simples para o equacionamento da altura de uma coluna de absorção dada pela Equação 13, em que o NUT pode ser calculado pela Equação 14.

$$
\begin{aligned}
\mathrm{Zt} & =\mathrm{HUT} . \mathrm{NUT} \\
N U T & =(y s-y e) / \ln \frac{(\mathrm{ys}-\mathrm{ys} *)}{(\mathrm{ye}-\mathrm{ye} *)}
\end{aligned}
$$

\subsection{Cálculo Do Diâmetro Da Coluna}

De acordo com Kohl e Nielsen (1997), um dos métodos possíveis para o dimensionamento do diâmetro de colunas de recheio é feito por meio da queda de pressão admissível com o intuito de evitar inundação, condição em que ocorre aumento da queda de pressão no sistema, perda de rendimento na absorção e aumento no consumo de energia para bombeamento do fluido. Falcão (2011), aborda que, usualmente, a literatura sugere que colunas sejam projetadas para operar com quedas de pressão entre 200 e $400(\mathrm{~Pa} / \mathrm{m}$ de coluna). Assim, a partir da Correlação de Sherwood determinou-se a perda de carga da coluna (FALCÃO, 2011) 


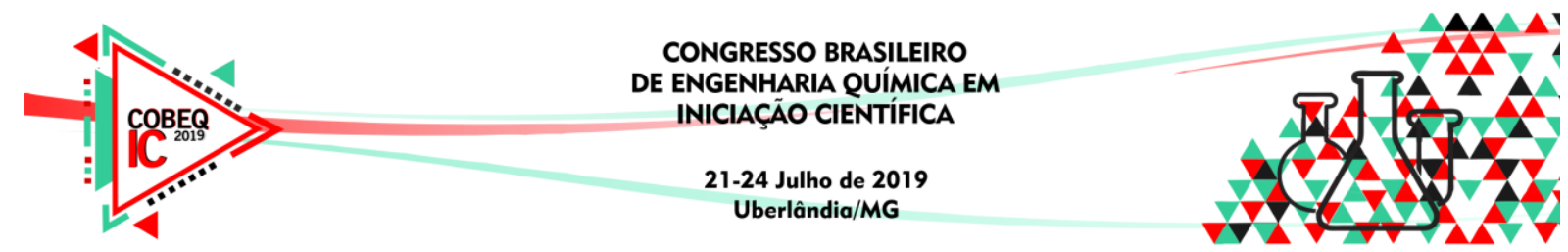

Dessa forma, pode-se obter a vazão de inundação “G”. Já a vazão de operação “GOP” é o equivalente a $40 \%$ da vazão de inundação para sistemas muito espumantes e $80 \%$ da vazão de inundação para sistemas pouco espumantes. Pode-se então, calcular o diâmetro da coluna de recheio por meio da Equação 15.

$$
\mathrm{DT}=\sqrt{\left.4 G^{\wedge^{\prime}} \pi G O P\right)}
$$

\subsection{Dimensões Da Coluna}

A partir da metodologia descrita e em comparação com outros trabalhos a coluna para absorção de $\mathrm{CO}_{2}$ com aminas poderá ter uma coluna de vidro com altura cerca de 1 metro e diâmetro de 0,3 metros. Além disso, é recomendado que a coluna tenha recheio estruturado a fim de reduzir quedas de pressão e proporcionar um escoamento mais uniforme da solução absorvedora, evitando acúmulos nas laterais do recipiente. Valores similares foram encontrados por Mello (2013) para dimensionamento de uma coluna piloto de pequeno porte

\section{CONCLUSÃO}

O presente trabalho apresenta o dimensionamento de uma coluna de absorção por meio de equações apresentadas na literatura, obtendo-se dimensões para a coluna quando soluções diferentes são empregadas no processo de captura de $\mathrm{CO}_{2}$. Verificou-se que a altura da coluna é mais influenciada pela concentração da solução absorvedora, enquanto o diâmetro é mais afetado pelos valores de vazão. Para escala laboratorial, espera-se trabalhar com uma coluna de absorção com cerca de 1 metro de altura e $0,3 \mathrm{~m}$ de diâmetro em que se possa variar as concentrações das soluções de MEA, AMP e PZ, bem como as vazões. Em trabalhos futuros espera-se realizar a montagem da coluna em conjunto com o recheio, e analisar o processo de absorção para captura de $\mathrm{CO}_{2}$ com as soluções aminas abordadas.

\section{NOMENCLATURA}

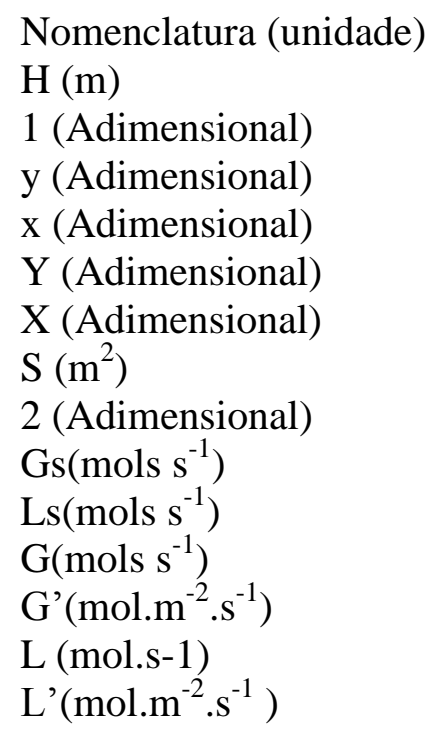

Grandeza

Altura do Enchimento

Base da Coluna

Fração Molar de Soluto Na Fase Gasosa

Fração Molar De Soluto Na Fase Líquida

Mol De Soluto/Mol De Gás

Mol De Soluto/Mol De Líquido

Seção Transversal Da Coluna

Topo Da Coluna

Vazão Molar De Gás Constante Ao Longo Da Coluna

Vazão Molar De Líquido Constante Ao Longo Da Coluna

Vazão Molar Molar De Gás Ao Longo Da Coluna

Vazão Molar Molar De Gás Por Unidade De Área (G/S)

Vazão Molar Molar De Líquido Ao Longo Da Coluna

Vazão Molar Molar De Líquido Por Unidade De Área (L/S) 


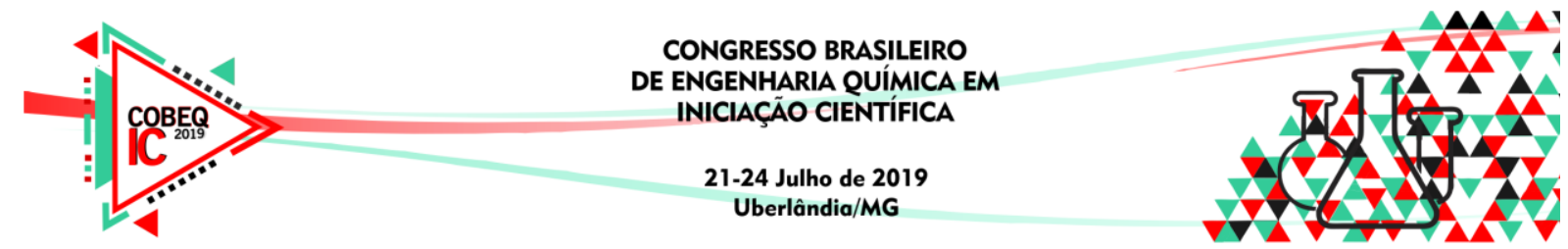

\section{REFERÊNCIAS}

FALCÃO, P. Equacionamento de torres de absorção. Dissertação (Trabalho de conclusão de curso) - Curso de Engenharia Química, Universidade Federal de Sergipe, São Cristóvão, 2011.

KOHL, A. L.; NIELSEN, R. Gas purification. Elsevier, 1997.

LAFER, M. Z. Estudo da absorção de $\mathrm{CO}_{2}$ em aminas: avaliação das demandas térmicas para uma coluna de escala industrial. Dissertação (Trabalho de conclusão curso) Curso de Engenharia Química, Universidade de São Paulo, São Paulo, 2011.

MELLO, L. C. Estudo do processo de absorção de CO2 em soluções de aminas empregando-se colunas recheadas. 2013. 125 f. Dissertação (Mestrado) - Curso de Engenharia Química, Universidade de São Paulo, São Paulo, 2013.

NASSER JUNIOR, Roberto. Otimização das colunas de absorção da recuperação de acetona na produção de FilterTow por meio de estudos fenomenológicos e análise estatística. Tese de Doutorado. Universidade de São Paulo. 2009.

Separações e operações unitárias. Disponível em: http://labvirtual.eq.uc.pt/siteJoomla/index.php?option=com_content\&task=view\&id=17 \&Itemid=135. Acesso em: 22 jun. 2018.

AROONWILAS, A. Effects of operating and design parameters on $\mathrm{CO} 2$ absorption in columns with structured packings. Separation and Purification Technology, v. 24, n. 3, p.403-411, 2001. 